\title{
ST Reha 1.0: Das neue Tarifsystem der stationären Rehabilitation
}

\author{
Bruno Trezzini ${ }^{\mathrm{a}}$, Beatrix Meyer ${ }^{\mathrm{b}}$ \\ ${ }^{a}$ Dr. phil., Experte, Abteilung Stationäre Versorgung und Tarife, FMH; ${ }^{b}$ Leiterin Abteilung Stationäre Versorgung und Tarife, FMH
}

Die schweizweit einheitliche Tarifstruktur ST Reha 1.0 liegt vor. Sie basiert auf leistungsbezogenen Tagespauschalen und soll per 1. Januar 2022 in Kraft treten. Zentrale Anliegen der FMH sind in die Tarifstruktur eingeflossen. Um die leistungsbezogene Abbildung aller Versorgungsmodelle zu gewährleisten, gilt es, eine Ergänzung zu den bestehenden rehaspezifischen CHOP-Kodes zu erarbeiten.

Nach langer Entwicklungszeit und der Überwindung zahlreicher Hürden ist es so weit: Der Verwaltungsrat der SwissDRG AG hat der Einführungsversion ST Reha 1.0 zugestimmt. Die Genehmigung durch den Bundesrat vorausgesetzt, wird ST Reha per 1. Januar 2022 in Kraft treten [1]. Nach der Einführung von SwissDRG in der Akutsomatik im Jahr 2012 und TARPSY in der Psychiatrie im Jahr 2018 kommen also auch in der Rehabilitation leistungsbezogene Pauschalen zur Anwendung. Damit werden die gesetzlichen Vorgaben gemäss Artikel 49 KVG umgesetzt. Die FMH und die betroffenen Fachgesellschaften haben die Entwicklung von ST Reha von Anfang an eng begleitet [2] und im letzten

Der Leistungsbezug sollte weiter verbessert werden, ohne zu einem überbordenden Dokumentationsaufwand zu führen.

Dezember auch zur Einführungsversion detailliert Stellung genommen. Die dort formulierten drei Vorbehalte der Fachgesellschaften zu ST Reha 1.0 konnten erfreulicherweise in Zusammenarbeit mit den Partnern weitgehend ausgeräumt werden. Im Folgenden beschreiben wir die wichtigsten Charakteristika von ST Reha 1.0 und heben die aus Sicht der FMH zentralen Entwicklungsschwerpunkte für künftige ST-Reha-Versionen hervor.

\section{Vom indirekten zum direkten Leistungsbezug}

Ursprünglich wurde bei der Entwicklung von ST Reha der Ansatz verfolgt, den Leistungsbezug indirekt abzubilden. Dies beinhaltete die Bestimmung des Leis- tungsbereichs (neurologische, kardiovaskuläre, pulmonale oder andere Funktionseinschränkungen) und die Erfassung des Schweregrads der Funktionseinschränkungen [3]. Eine direkte Erfassung der Behandlungsintensität war nicht vorgesehen. Am 1. Juni 2018 entschied der Verwaltungsrat der SwissDRG AG jedoch, dass ST Reha durch den zusätzlichen Einbezug von Kodes der Prozedurenklassifikation CHOP einen direkten Leistungsbezug erhalten soll [4].

\section{Definierte Basis- und Zusatzleistungen als zentrale Grundlage}

Bereits im Vorfeld engagierte sich die FMH zusammen mit den Fachgesellschaften, um die Basis- und Zusatzleistungen von acht Rehabilitationsarten [5] zu definieren. Basisleistungen umfassen dabei all jene Leistungen, welche jeder Patient für eine qualitativ hochstehende Rehabilitation mindestens erhalten soll. Zusatzleistungen werden je nach Indikation und Bedarf ergänzend erbracht, um eine an die Patientenbedürfnisse optimal angepasste Behandlung zu gewährleisten [6]. Diese umfangreichen Arbeiten der FMH und ihrer Fachgesellschaften sind weitgehend in die CHOP eingeflossen, auch wenn Kompromisse gegenüber den Partnern der SwissDRG AG erforderlich waren. Geschaffen wurden damit die CHOP-Kodes BA.1 bis BA.8 für die Basisleistungen der acht Rehabilitationsarten und der BB.1-CHOP-Kode für indikationsbezogene Zusatzleistungen [7]. Die in den Basisleistungen enthaltenen Definitionen von therapeutischen Mindestschwellen haben im Vorfeld teilweise zu Missverständnissen geführt. So wurden Befürchtungen laut, dass Versorgungsmodelle mit einer eher pflegeorientierten Reha- 
bilitation beispielsweise für geriatrische Patienten von ST Reha ausgeschlossen werden könnten [8]. Dies trifft jedoch nicht zu. Ausserdem bestand die Sorge, dass es zu einem Leistungsabbau und einer Qualitätseinbusse kommen könnte, falls wichtige therapeutische Leistungen über der Mindestschwelle nicht mehr vergütet würden. Dieses Problem konnte jedoch inzwischen

Tabelle 1: Reha Cost Groups (RCGs) und deren Tageskostengewichte (TKG).

\begin{tabular}{|c|c|c|}
\hline RCG & Bezeichnung RCG & TKG \\
\hline \multicolumn{3}{|c|}{ TR11 Rehabilitation für Kinder und Jugendliche } \\
\hline TR11A & $\begin{array}{l}\text { Rehabilitation für Kinder und Jugendliche, Alter <19 Jahren, } \\
\text { mit komplizierender Diagnose }\end{array}$ & 1.962 \\
\hline TR11B & Rehabilitation für Kinder und Jugendliche, Alter $<16$ Jahren & 1.752 \\
\hline TR11C & Rehabilitation für Kinder und Jugendliche & 1.173 \\
\hline \multicolumn{3}{|c|}{ TR13 Neurologische Rehabilitation } \\
\hline TR13A & $\begin{array}{l}\text { Aufwendige neurologische Rehabilitation mit komplizierender } \\
\text { Diagnose oder mit hohem Zusatzaufwand }\end{array}$ & 1.348 \\
\hline TR13B & $\begin{array}{l}\text { Neurologische Rehabilitation mit komplizierender Diagnose } \\
\text { oder mit Zusatzaufwand oder mit bestimmter Behandlung }\end{array}$ & 1.114 \\
\hline TR13C & Neurologische Rehabilitation & 1.007 \\
\hline \multicolumn{3}{|c|}{ TR14 Internistische oder onkologische Rehabilitation } \\
\hline TR14A & $\begin{array}{l}\text { Aufwendige internistische oder onkologische Rehabilitation } \\
\text { mit komplizierender Diagnose }\end{array}$ & 1.191 \\
\hline TR14B & $\begin{array}{l}\text { Internistische oder onkologische Rehabilitation mit bestimm- } \\
\text { ter Funktionseinschränkung }\end{array}$ & 1.045 \\
\hline TR14C & Internistische oder onkologische Rehabilitation & 0.982 \\
\hline \multicolumn{3}{|c|}{ TR15 Geriatrische Rehabilitation } \\
\hline TR15A & $\begin{array}{l}\text { Aufwendige geriatrische Rehabilitation mit komplizierender } \\
\text { Diagnose oder mit bestimmter Behandlung }\end{array}$ & 1.072 \\
\hline TR15B & Geriatrische Rehabilitation & 0.973 \\
\hline \multicolumn{3}{|c|}{ TR16 Muskuloskelettale Rehabilitation } \\
\hline TR16A & $\begin{array}{l}\text { Aufwendige muskuloskelettale Rehabilitation mit kompli- } \\
\text { zierender Diagnose oder mit bestimmter Behandlung }\end{array}$ & 1.077 \\
\hline TR16B & $\begin{array}{l}\text { Muskuloskelettale Rehabilitation mit bestimmter Funktions- } \\
\text { einschränkung }\end{array}$ & 0.933 \\
\hline TR16C & Muskuloskelettale Rehabilitation & 0.898 \\
\hline \multicolumn{3}{|c|}{ TR17 Pulmonale Rehabilitation } \\
\hline TR17A & $\begin{array}{l}\text { Aufwendige pulmonale Rehabilitation mit begrenzter } \\
\text { Leistungsfähigkeit oder mit bestimmter Diagnose oder } \\
\text { mit bestimmter Behandlung }\end{array}$ & 1.091 \\
\hline TR17B & Pulmonale Rehabilitation & 0.979 \\
\hline \multicolumn{3}{|c|}{ TR18 Psychosomatische Rehabilitation } \\
\hline TR18Z & Psychosomatische Rehabilitation & $\begin{array}{l}1.302^{*} \\
0.838^{* *} \\
0.630^{* * *}\end{array}$ \\
\hline \multicolumn{3}{|c|}{ TR19 Kardiale Rehabilitation } \\
\hline TR19A & $\begin{array}{l}\text { Aufwendige kardiale Rehabilitation mit bestimmter } \\
\text { Funktionseinschränkung }\end{array}$ & 0.900 \\
\hline TR19B & Kardiale Rehabilitation & 0.782 \\
\hline \multicolumn{3}{|c|}{ TR80 Rehabilitation ohne weitere Angabe } \\
\hline TR80Z & Rehabilitation ohne weitere Angabe & 0.782 \\
\hline \multicolumn{3}{|c|}{ TR96 Nicht gruppierbar } \\
\hline TR96Z & Nicht gruppierbar & - \\
\hline
\end{tabular}

* von Aufenthaltstag 1 bis $3 ; * *$ von Aufenthaltstag 4 bis $25 ; * * *$ ab Aufenthaltstag 26.

Quelle: Basierend auf SwissDRG AG, RCG-Katalog ST Reha 1.0, 12.3.2021 (www.swissdrg.ch

$\rightarrow$ Rehabilitation $\rightarrow$ ST Reha System 1.0/2022 $\rightarrow$ RCG-Katalog). mit der Schaffung des zusätzlichen BB.2-CHOP-Kodes entschärft werden. Mit diesen Kodes ist es seit Anfang 2021 möglich, die erbrachten Therapieleistungen oberhalb der definierten Mindestschwellen abgestuft zu erfassen.

\section{ST Reha 1.0 kurz erklärt}

Die SwissDRG AG hat ST Reha 1.0 basierend auf den Leistungs- und Kostendaten des Jahres 2019 von 65 Kliniken kalkuliert [9]. Insgesamt 59585 plausible Patientenfälle gingen in die Kalkulation ein, wovon $72 \%$ auf die Alterskategorie 65 Jahre oder älter entfielen. Die einzelnen Fälle werden anhand eines Patientenklassifikationsalgorithmus (Grouper) verschiedenen Rehabilitations-Kostengruppen (RCGs) zugeteilt. Im Grouper kommen die folgenden kostentrennenden Gruppierungskriterien zur Anwendung: (1) die Basisleistungen in der Rehabilitation (BA-Kodes); (2) die Analogiekodierung der ursprünglichen vier Leistungsbereiche (neurologische, kardiovaskuläre, pulmonale und andere Funktionseinschränkungen [10]); (3) Zusatzleistungen in der Rehabilitation (wie der BB.1-Kode oder eine Berufsrehabilitation); (4) Indikatoren für das Ausmass von kognitiven oder motorischen Funktionseinschränkungen; (5) aufwendige Diagnosen und (6) das Alter. ST Reha 1.0 umfasst neun Basis-RCGs, welche in bis zu drei Untergruppen aufgeteilt und mit unterschiedlichen Tageskostengewichten hinterlegt sind (Tab. 1). Acht Basis-RCGs entsprechen dabei den acht Rehabilitationsarten. In diese acht Basis-RCGs wird ein Fall einerseits mittels der CHOP-Kodes der Basisleistungen BA.1 bis BA.8 zugeteilt. Wurde keiner der Kodes BA.1 bis BA.8 kodiert, kann der Fall andererseits anhand der Analogiekodierung in eine dieser acht BasisRCGs gelangen [11]. Die neunte Basis-RCG (TR80) repräsentiert die Restgruppe «Rehabilitation ohne weitere Angabe».

Insgesamt besteht die Tarifstruktur aus 21 RCGs. Wie schon im Falle von TARPSY hatten die Analysen der SwissDRG AG ergeben, dass Tagespauschalen geeigneter für die Tarifierung der stationären Rehabilitation sind als Fallpauschalen. In der Regel sind die anwendbaren Tageskostengewichte über einen ganzen Rehabilitationsaufenthalt hinweg konstant. Einzig bei der RCG TR18Z (psychosomatische Rehabilitation) ist die Aufenthaltsdauer in unterschiedlich kostenintensive Phasen unterteilt (Tab. 1). Der Erlös für die erbrachten Rehabilitationsleistungen ergibt sich schlussendlich aus der Multiplikation der Tageskostengewichte mit der Anzahl Aufenthaltstage des Patienten und dem ausgehandelten Basispreis. Mit der Tarifstruktur allein können jedoch nicht alle Kostenunterschiede zwi- 
schen Spitälern erklärt werden; regionale Lohnunterschiede oder spital- und klinikindividuelle Strukturmerkmale sind beispielsweise nicht darin abgebildet [12]. Auch gilt es bei der Systemumstellung zu beachten, dass gewisse Leistungen, z.B. therapeutische Belastungserprobungen, im neuen System anders behandelt werden (nämlich als urlaubsbedingte Abwesenheit) und deshalb eine geringere Vergütung resultiert als im alten System. Solche Aspekte sind bei den Tarifverhandlungen zu berücksichtigen.

\section{Wichtige Anliegen der Fachgesellschaften wurden aufgenommen}

Der Grouper von ST Reha 1.0 weist Unzulänglichkeiten auf, welche Fehlanreize, eine Verwässerung des Leistungsbezugs oder einen vermehrten administrativen Aufwand nach sich ziehen können. Deshalb unterbreitete die FMH in ihrer Stellungnahme zu ST Reha 1.0 der SwissDRG AG verschiedene Anpassungs- und Vorgehensvorschläge. Im weiteren Verlauf der Vernehmlassung und nach verschiedenen Gesprächen zwischen den Partnern wurden die Anliegen der FMH und der

\section{Über ST Reha müssen sich unterschiedliche Versorgungsmodelle sachgerecht abbilden und vergüten lassen.}

betroffenen Fachgesellschaften erfreulicherweise weitestgehend berücksichtigt. So nahm die SwissDRG AG beispielsweise einen normativen Eingriff vor, um zu gewährleisten, dass das Tageskostengewicht der Restgruppe TR80Z nicht höher ausfällt als die Tageskostengewichte aller anderen RCGs. Des Weiteren wird die behelfsmässige Verwendung von Analogiekodierungen anstelle der bestehenden rehaspezifischen CHOPKodes nur bis maximal zur Datenerhebung 2024 (Daten 2023) erlaubt sein. Dies ist deshalb wichtig, weil die Analogiekodierungen über keinen expliziten Leistungsbezug verfügen. Schliesslich bestand auch die Befürchtung, im Falle von Patienten, die jünger als 19 oder älter als 79 Jahre sind, mit zusätzlichem administrativem Aufwand oder gar einem Erlösausfall konfrontiert zu werden. Dies deshalb, weil bei solchen Patienten die Zuordnung zu einer spezifischen BasisRCG am Ende der Behandlung unter Umständen vom Leistungsauftrag der Klinik oder der ursprünglichen Kostengutsprache abweichen kann [13]. Die Gesundheitsdirektorenkonferenz (GDK) hat auf Anregung der FMH dankenswerterweise ein klärendes Schreiben zum kantonalen Leistungscontrolling unter ST Reha verfasst, das diese Problematik entschärft. Die GDK verweist u.a. darauf, dass die Kantone das Leistungscontrolling nicht aufgrund der Rechnung, sondern aufgrund der erbrachten Leistungen vornehmen.

\section{Abbildung der pflegeorientierten Rehabilitation vorantreiben}

Aus einer Reihe von Entwicklungsschwerpunkten für künftige ST-Reha-Versionen, welche die FMH zuhanden der SwissDRG AG zusammengestellt hat, sollen im Folgenden drei hervorgehoben werden: die Ablösung der Analogiekodierung, die Schaffung rehaspezifischer Zusatzentgelte z.B. für teure Medikamente sowie Massnahmen zur Verbesserung der Datenqualität [14] Durch die Analogiekodierung kommt es zu einer Verwässerung des geforderten Leistungsbezugs und zu Fehlanreizen [15]. Deshalb ist eine rasche Ablösung der Analogiekodierung wichtig. Der geeignetste Weg, um dies zu erreichen, ist aus Sicht der FMH und ihrer Fachgesellschaften die Schaffung eines spezifischen CHOP-Kodes für die pflegeorientierte Rehabilitation. Dadurch würde sowohl den Ansprüchen an eine qualitativ hochstehende Rehabilitation als auch der Realität unterschiedlicher Versorgungsstrukturen adäquat Rechnung getragen. Demgegenüber haben sich die FMH und ihre Fachgesellschaften entschieden gegen eine allfällige Absenkung der bestehenden Mindestschwellen bei den therapeutischen Leistungen ausgesprochen

\section{Anträge für rehabilitationsspezifische Zusatzentgelte in Arbeit}

Die im Zusatzentgelt-Katalog [16] der Akutsomatik aufgeführten Leistungen dürfen zusätzlich zur ST-RehaPauschale abgerechnet werden. Zu nennen sind hier beispielsweise teure Medikamente oder Verfahren wie die Dialyse. Teilweise handelt es sich dabei um Leistungen, die klinikextern erbracht werden. Es ist zwar positiv, dass die Zusatzentgelte der Akutsomatik auch für ST Reha 1.0 gültig sind. Für künftige ST-Reha-Versionen ist jedoch zu prüfen, welche rehabilitationsspezifischen Zusatzentgelte es zusätzlich braucht. Auf Anregung von Spitalvertretenden hat $\mathrm{H}+$ deshalb bei den Rehabilitationskliniken Informationen zu kostenintensiven Medikamenten gesammelt. Basierend darauf wird die FMH zusammen mit den Fachgesellschaften im Rahmen des diesjährigen Antragsverfahrens [17] entsprechende Anträge für die Schaffung von Zusatzentgelten oder für die Aufnahme auf die Liste der in der Medizinischen Statistik erfassbaren Medikamente/ Substanzen stellen. 


\section{Datenqualität verbessern}

Etwas mehr als ein Fünftel der für das Jahr 2019 gelieferten Fälle erwies sich gemäss der SwissDRG AG als unplausibel und musste von den Kalkulationen ausgeschlossen werden. Die SwissDRG AG hat deshalb bereits den Dialog mit den Kliniken gesucht und Massnahmen zur Verbesserung der Datenqualität eingeleitet, z.B. Plausibilitätstests und Einzelfallprüfungen. Die FMH regt ferner an, zusätzlich eine konkrete Massnahmenliste zur Verbesserung der Datenqualität zu erarbeiten. Hinsichtlich der Kodierung der BA.- und BB.-CHOPKodes gilt es zudem sicherzustellen, dass die Mindestanforderungen effektiv erfüllt wurden. Hier kommt der Kodierrevision eine entscheidende Bedeutung zu [18]. Zur einfacheren Erfassung der Basis- und Zusatzleistungen in der Rehabilitation bzw. der BA.- und BB.CHOP-Kodes stellt die FMH ihren Mitgliedern Dokumentationsmuster zur Verfügung [19]. Darüber hinaus dürften sich die Daten der Jahre 2020 und 2021 aufgrund der Corona-Pandemie und der von den Behörden eingeleiteten Massnahmen von jenen des Jahres 2019 unterscheiden. Die SwissDRG AG wird deshalb analysieren, inwieweit die Daten 2020 für die Tarifstrukturentwicklung verwendet werden können, mit welchen allfälligen Verzerrungen $\mathrm{zu}$ rechnen ist und ob eine Bereinigung um den Corona-Effekt möglich wäre.

\section{Tarifautonomie hat sich bewährt}

ST Reha 1.0 stärkt die Tarifautonomie und ist ein weiterer Erfolg für die tarifpartnerschaftliche Zusammenarbeit im schweizerischen Gesundheitswesen. ST Reha 1.0 stellt zwar in vielerlei Hinsicht einen Kompromiss dar. Aber wie die bereits bestehenden leistungsbezogenen Tarifsysteme in der Akutsomatik und der Psychiatrie ist auch ST Reha 1.0 als lernendes System konzipiert. Die Grundlage von ST Reha bilden u.a. die in der CHOP beschriebenen Basis- und Zusatzleistungen der eher therapieorientierten Rehabilitation. Um den unterschiedlichen in der Schweiz bestehenden Versorgungsmodellen [20] gerecht zu werden, gilt es nun, die eher pflegeorientierte Rehabilitation ebenfalls in der CHOP abzubilden. Die FMH wird sich zusammen mit den Fachgesellschaften weiterhin aktiv für eine Weiterentwicklung von ST Reha in diesem Bereich engagieren.

Abteilung Stationäre

Versorgung und Tarife Baslerstrasse 47

CH-4600 Olten

Tel. 0313591111

Fax 0313591112

tarife.spital[at]fmh.ch
3 Ingenpass P, Rohner B. ST Reha - Leistungsbezogene Tagespauschalen. Schweiz Ärzteztg. 2015;96(18):634-5.

4 SwissDRG AG. SwissDRG AG verschiebt Einführung der Tarifstruktur für die stationäre Rehabilitation (ST Reha). 1.6.2018 (www.swiss drg.ch $\rightarrow$ Über uns $\rightarrow$ Verwaltungsrat $\rightarrow$ Kommunikation). Meyer B. ST Reha: Richtungswechsel in der Tarifstruktur. Schweiz Ärzteztg. 2018;99(30-31):966-7.

5 Neurologische, psychosomatische, pulmonale, kardiale, muskuloskelettale, internistische/onkologische, pädiatrische und geriatrische Rehabilitation.

6 www.fmh.ch $\rightarrow$ Themen $\rightarrow$ Stationäre Versorgung $\rightarrow$ ST Reha

7 Trezzini B, Meyer B. Weiterer Meilenstein auf dem Weg zu ST Reha erreicht. Schweiz Ärzteztg. 2019;100(6):161-2.

8 Plateforme-reha.ch. Reha für ältere Menschen in Gefahr, 28.2.2020 (www.plateforme-reha.ch $\rightarrow$ Presse \& Links).

9 SwissDRG AG. Folien ST Reha 1.0 Systempräsentation, 14.12.2020 (www.swissdrg.ch $\rightarrow$ Rehabilitation $\rightarrow$ ST Reha System 1.0/2022 $\rightarrow$ Ergänzende Dokumente).

10 Beim Leistungsbereich «andere Funktionseinschränkung» kommt jeweils zusätzlich die Hauptdiagnose zur Anwendung.

11 Beispiel: In ST Reha 1.0 kann ein Fall nicht nur über den BA.1-CHOPKode (neurologische Rehabilitation) in die Basis-RCG TR13 (neurologische Rehabilitation) gelangen, sondern auch über die Kodierung «Leistungsbereich Neurologie» sowie über die Kodierung "andere» im Verbund mit einer neurologischen Diagnose.

12 SwissDRG AG. Abbildung von Kostenunterschieden durch die Tarifstruktur ST Reha und Schlussfolgerungen für differenzierte Preise, 12.3.2021 (www.swissdrg.ch $\rightarrow$ Über uns $\rightarrow$ Verwaltungsrat $\rightarrow$ Kommunikation).

13 Ein siebzehnjähriger Patient, der beispielsweise eine muskuloskelettale Rehabilitation erhält, wird vom Grouper nicht der Basis-RCG TR16 (muskuloskelettale Rehabilitation) zugeteilt, sondern immer der Basis-RCG TR11 (Rehabilitation für Kinder und Jugendliche). Die Zuordnung von Patienten, die älter als 79 sind, kann unter gewis sen Konstellationen von ähnlichen begrifflichen Diskrepanzen betroffen sein.

14 Weitere von der FMH empfohlene Schwerpunkte betreffen die folgenden Aspekte: die Verwendung mehrerer Datenjahre für RCGs mit kleinen Fallzahlen; die Bereitstellung eines Datenspiegels und von Informationen zur Kostenhomogenität der RCGs; die Gewährleistung eines ausgewogenen Verhältnisses zwischen Aufwand und Ertrag in der Leistungsdokumentation; die systematische Überprüfung verschiedener Zusatzaufwand-Niveaus auf ihre Eignung als Kostentrenner; die Überprüfung des kostentrennenden Effekts der Cumulative Illness Rating Scale (CIRS) und des Vorhandenseins mehrerer komplizierender Nebendiagnosen; die leistungsgerechte Vergütung der Belastungserprobung auch im neuen Tarifsystem; die Erforschung der Auswirkungen des mit ST Reha 1.0 einhergehenden Systemwechsels; die langfristige Regelung der Vergütung von Fällen in der Paraplegiologie und der Frührehabilitation.

15 Bei denjenigen Basis-RCGs, die über mehrere Untergruppen verfügen, betrifft die Verwässerung des geforderten Leistungsbezugs jeweils die Stufen B und C. Die Stufe A, die jeweils das höchste Tageskostengewicht aufweist, ist davon nicht betroffen, da für die Zuteilung eines Falls in diese Untergruppe ein BA-Kode Voraussetzung ist.

16 Der Zusatzentgelt-Katalog der Akutsomatik ist unter www.swissdrg.org in den Anlagen 2 und 3 des Fallpauschalenkatalogs der jeweils aktuellen SwissDRG-Version zu finden.

17 www.swissdrg.ch $\rightarrow$ Rehabilitation $\rightarrow$ Antragsverfahren

18 www.swissdrg.ch $\rightarrow$ Rehabilitation $\rightarrow$ ST Reha System 1.0/2020 $\rightarrow$ Kodierrevision

19 www.fmh.ch $\rightarrow$ Themen $\rightarrow$ Stationäre Tarife $\rightarrow$ ST Reha

20 Diese systematischen Unterschiede in den Versorgungsmodellen und ihr Bezug zum Thema Rehabilitation werden in einer kürzlich vom Schweizerischen Gesundheitsobservatorium publizierten Studie besonders deutlich; siehe Füglister-Dousse S, Pellegrini S. Hospitalisierungen von Betagten im letzten Lebensjahr. Verbleib zuhause versus Heimaufenthalt. Neuenburg: Schweizerisches Gesundheitsobservatorium; Obsan Bulletin 04/2021. 